\title{
Non-invasive surveys of mammalian viruses using environmental DNA
}

\author{
Niccolò Alfano ${ }^{1,2}$ (i) | Anisha Dayaram ${ }^{3,4}$ (i) | Jan Axtner ${ }^{1}$ (i) | Kyriakos Tsangaras ${ }^{5}$ \\ Marie-Louise Kampmann $^{1,6}$ (D) | Azlan Mohamed ${ }^{1,7}$ | Seth T. Wong ${ }^{1}$ | \\ M. Thomas P. Gilbert ${ }^{8,9}$ (i) | Andreas Wilting ${ }^{1}$ (D) | Alex D. Greenwood ${ }^{3,10}$
}

\begin{abstract}
${ }^{1}$ Department of Ecological Dynamics, Leibniz Institute for Zoo and Wildlife Research, Berlin, Germany; ${ }^{2}$ Department of Biology and Biotechnology, University of Pavia, Pavia, Italy; ${ }^{3}$ Department of Wildlife Diseases, Leibniz Institute for Zoo and Wildlife Research, Berlin, Germany; ${ }^{4}$ Charité-Universitätsmedizin Berlin, Corporate Member of Freie Universitäts Berlin and Humboldt-Universität of Berlin, Institut für Neurophysiologie, Berlin, Germany; ${ }^{5}$ Department of Life and Health Sciences, University of Nicosia, Nicosia, Cyprus; ${ }^{6}$ Section of Forensic Genetics, Department of Forensic Medicine, Faculty of Health and Medical Sciences, University of Copenhagen, Copenhagen, Denmark; ${ }^{7}$ WWF-Malaysia, PJCC, Petaling Jaya, Malaysia; ${ }^{8}$ The GLOBE Institute, University of Copenhagen, Copenhagen, Denmark; ${ }^{9}$ University Museum, NTNU, Trondheim, Norway and ${ }^{10}$ Department of Veterinary Medicine, Freie Universität Berlin, Berlin, Germany
\end{abstract}

Correspondence

Andreas Wilting

Email: wilting@izw-berlin.de

Alex D. Greenwood

Email: greenwood@izw-berlin.de

Funding information

Bundesministerium für Bildung und Forschung, Grant/Award Number: 01LN1301A; Leibniz-Gemeinschaft, Grant/ Award Number: SAW-2015-IZW-1 440

Handling Editor: Robert Freckleton

\section{Abstract}

1. Environmental DNA (eDNA) and invertebrate-derived DNA (iDNA) are used to survey biodiversity non-invasively to mitigate difficulties in obtaining wildlife samples, particularly in remote areas or for rare species. Recently, eDNA/iDNA were used to monitor known wildlife pathogens; however, most wildlife pathogens are unknown and often evolutionarily divergent.

2. To detect and identify known and novel mammalian viruses from eDNA/iDNA, we used a curated set of RNA oligonucleotides as viral baits in a hybridization capture system coupled with high-throughput sequencing.

3. We detected multiple known and novel mammalian RNA and DNA viruses from multiple viral families from both waterhole eDNA and leech-derived iDNA. Congruence was found between detected hosts and viruses identified in leeches and waterholes.

4. Our results demonstrate that eDNA/iDNA samples represent an effective noninvasive resource for studying wildlife viral diversity and for detecting novel potentially zoonotic viruses prior to their emergence.

\section{KEYWORDS}

environmental DNA (eDNA), hybridization capture, leeches, non-invasive samples, viral diversity 


\section{1 | INTRODUCTION}

Emerging infectious viruses increasingly threaten human, domestic animal and wildlife health (Johnson et al., 2019). Sixty percent of emerging infectious diseases in humans are of zoonotic origin (Jones et al., 2008). Wildlife trade and consumption of bushmeat, especially in Africa and Asia, play a role in zoonotic emergence (Pruvot et al., 2019; Swift et al., 2007). Wildlife markets may have facilitated the spillover of pandemic SARS-CoV-2 to humans (Peeri et al., 2020; Zhou et al., 2020). The 2002-2003 SARS-CoV outbreak (Drosten et al., 2003), the Ebola outbreak in West Africa (Leroy et al., 2009) and the global emergence of HIV (Sharp \& Hahn, 2011) were all linked to wildlife trade and bushmeat consumption. Early detection of novel infectious agents in wildlife is key to emergence prevention. However, identification, surveillance and monitoring of emerging viruses by directly sampling wildlife often require enormous sampling investment, particularly for viruses that have low prevalence (Hoye et al., 2010). For example, 25,000 wild birds were sampled in Germany to detect avian influenza prevalence below 1\% (Wilking et al., 2009). Sampling over 8,157 animals in Poland was required to detect African swine fever virus with a $0.12 \%$ prevalence (Śmietanka et al., 2016). Developing viral surveillance and discovery methods remains challenging and is often hindered by lack of access to free ranging wildlife.

Non-invasive nucleic acid sources, such as environmental DNA (eDNA) and invertebrate-derived DNA (iDNA), can mitigate difficulties in obtaining wildlife samples under remote field conditions to complement invasive sampling or replace it when invasive sampling is not possible. eDNA and iDNA have been mainly used to survey biodiversity, for instance water eDNA has been used to survey mammalian biodiversity in African waterholes (Seeber et al., 2019) and terrestrial haematophagous leeches have been used to survey Southeast Asian mammals (Abrams et al., 2019; Schnell et al., 2018; Tilker et al., 2020). Water is ubiquitous in most ecosystems, and, among invertebrates, leeches are abundant and easy to collect in many tropical rainforests, many of which are major hotspots of emerging infectious diseases (Daszak et al., 2000). Recently, eDNA/iDNA have been applied to monitoring specific wildlife pathogens (Gogarten et al., 2019; Mosher et al., 2017). However, previous studies focused on known pathogens, whereas most wildlife pathogens are uncharacterized, particularly in tropical emerging virus hotspots (Jones et al., 2008). To identify and characterize novel mammalian viruses from eDNA (waterhole water and sediment from Tanzania and Mongolia) and iDNA (haematophagous terrestrial leeches from Malaysia), we designed a hybridization capture system using a curated set of RNA oligonucleotides based on the ViroChip microarray assay (Chen et al., 2011) as baits to comprehensively target mammalian viral genomes (Figure 1). Multiple known and novel viruses were identified from both eDNA and iDNA samples including a novel divergent coronavirus demonstrating that eDNA/iDNA samples can be used to survey known and unknown viral diversity.

\section{2 | MATERIALS AND METHODS}

\section{1 | Sample collection}

\subsection{1 | Leeches}

Two types of terrestrial leeches, tiger leeches Haemadipsa picta and brown leeches Haemadipsa zeylanica, were collected from February to May 2015 in the Deramakot Forest Reserve in Sabah, Malaysian Borneo as described in Axtner et al. (2019). All leeches of the same species (tiger or brown) and from the same sampling site and time were pooled and processed as one sample. The number of leeches ranged from 1 to 77 per pool (median = 7). Samples were stored in RNAlater ${ }^{\mathrm{TM}}$ and exported under the permit 'JKM/MBS.1000-2/3 JLD.2 (8)'. A total of 68 pools (L1-L68) were selected for viral capture to maximize representation of host wildlife species identified from bloodmeals (Axtner et al., 2019).

\subsection{2 | Sediment and water}

In February, June, July and October 2016, samples were collected from the Serengeti National Park (c. $2.2^{\circ} \mathrm{S}, 34.8^{\circ} \mathrm{E}$ ) Tanzania from waterholes. In October 2015, samples were collected from Southeast Gobi $\left(45.5905^{\circ} \mathrm{N}, 107.1596^{\circ} \mathrm{E}\right)$ and in between June and July 2016 samples were collected from Gobi $B\left(45.1882^{\circ} \mathrm{N}, 93.4288^{\circ} \mathrm{E}\right)$ in Mongolia. At each waterhole, $50 \mathrm{ml}$ of water was passed through a 0.22- $\mu \mathrm{m}$ Sterivex filter unit (Millipore) using a disposable $50-\mathrm{ml}$ syringe to remove debris from water. In addition, $25 \mathrm{~g}$ of the top 1-3 cm of sediment was collected at each waterhole. The samples were stored on ice in the field, and then frozen at $-20^{\circ} \mathrm{C}$. In total, water filtrate and sediment samples were sampled at 12 waterholes, six, respectively, from Mongolia and Tanzania. For each sample, $32 \mathrm{ml}$ of water filtrate was ultra-centrifuged at $28,000 \mathrm{rpm}$ for $2 \mathrm{hr}$ to pellet DNA and viral particles. The supernatant was then removed, the pellet re-suspended in $1 \mathrm{ml}$ of cold PBS (pH 7.2; Sigma-Aldrich) and left at $4^{\circ} \mathrm{C}$ overnight.

\section{2 | Preparation of samples and nucleic acid extraction}

\subsection{1 | Leeches}

Leeches were cut into small pieces with a scalpel blade and lysed overnight ( $\geq 12 \mathrm{hr}$ ) at $55^{\circ} \mathrm{C}$ in proteinase $\mathrm{K}$ and ATL buffer at a 1:10 ratio; $0.2 \mathrm{ml}$ per leech. Nucleic acids were extracted from leech samples using the DNeasy 96 Blood \& Tissue kit (Qiagen; see Axtner et al., 2019).

\subsection{2 | Water and sediment}

$500 \mu$ of the centrifuged filtrate was used to extract viral nucleic acids using the RTP ${ }^{\circledR}$ DNA/RNA Virus Mini Kit (Stratec biomedical) 


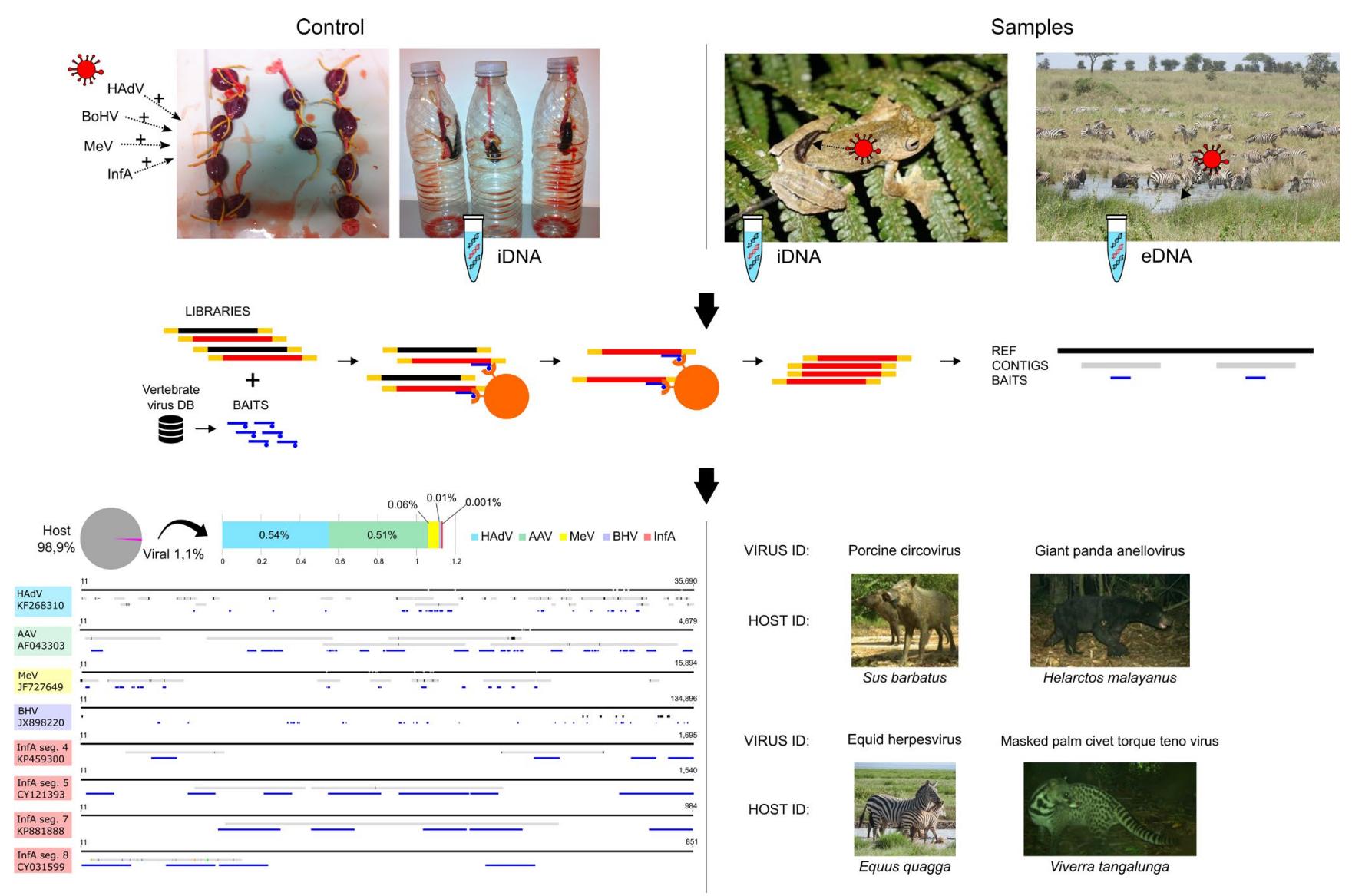

FIGURE 1 Viral screening of vertebrate viruses from leech positive control and leech iDNA and waterholes eDNA samples using hybridization capture. Left panel: medical leeches which were fed human blood spiked with four viruses were used as positive controls. All four viruses were detected after capture, with different percentages of on-target viral reads. The contigs recovered for each virus (grey/black bars) were mapped to the reference genome together with the corresponding baits (blue bars). Right panel: examples of iDNA and eDNA samples: a leech feeding on a frog in a rainforest of Vietnam (courtesy Andrew Tilker; Leibniz-IZW) and an African waterhole in Tanzania (courtesy Peter Seeber; Leibniz-IZW). Viral identity of contigs found in eDNA and iDNA samples is paired with host identity determined either by mammalian metabarcoding of the leech samples, or by observation of waterhole usage

with the following modifications: $400 \mu$ l of lysis buffer, $400 \mu \mathrm{l}$ of binding buffer and $20 \mu \mathrm{l}$ of proteinase $\mathrm{K}$ and carrier RNA were used per sample. Samples were eluted in $60 \mu \mathrm{l}$. The NucleoSpin Soil kit (Macherey-Nagel) was used to extract DNA/RNA from sediment. $500 \mathrm{mg}$ of soil was extracted according to the manufacture's protocol using a $100 \mu$ l elution volume.

\section{3 | Positive control}

Positive control medical leeches (Hirudo spp.) were fed human blood spiked with four viruses at different concentrations (Kampmann et al., 2017). Two RNA viruses, influenza A (InfA) and measles morbillivirus (MeV), and two DNA viruses, bovine herpesvirus (BHV) and human adenovirus (HAdV), were used. The nucleic acids were extracted from the leeches 7 days after feeding and the viral concentrations measured by qPCR (see Kampmann et al., 2017).

\section{4 | Library preparation}

RNA was reverse transcribed using SuperScript III and IV (Thermo Fisher Scientific) with random hexamers prior to second-strand synthesis with Klenow fragment (New England Biolabs). The resulting double-stranded cDNA/DNA mix was sheared to an average fragment size of 200 bp using an M220 focused ultrasonicator (Covaris). Sheared product was purified using the ZR-96 DNA Clean \& Concentrator-5 kit (Zymo). Dual-indexed Illumina sequencing libraries were constructed as described by Meyer and Kircher (2010) with the modifications described in Alfano et al. (2016). Each library was amplified in three replicate reactions to minimize amplification bias of individual PCRs. The three replicate PCR products for each sample were pooled and purified using the MinElute PCR Purification Kit (Qiagen). Negative control libraries were prepared from different stages of the experimental process (extraction, reverse transcription, library preparation and index PCR) and indexed separately to monitor for contamination. Amplified libraries were 
quantified using the 2200 TapeStation (Agilent Technologies) on D1000 ScreenTapes.

\section{5 | RNA oligonucleotide bait design}

The targeted sequence capture panel was designed based on the oligonucleotide probes represented on the Virochip microarray (Wang et al., 2002). The Virochip is a pan-viral DNA microarray comprising the most highly conserved 70-mer sequences from fully sequenced reference viral genomes in GenBank, which was developed for the rapid identification and characterization of novel viruses. We retrieved the viral oligonucleotides

from the fifth-generation Virochip (Viro5; Yozwiak et al., 2012), which are available at NCBI's Gene Expression Omnibus (GEO) repository, (https://www.ncbi.nlm.nih.gov/geo/query/acc.cgi?acc=GPL13323). This included $~ 17,500$ oligonucleotides (70-mer nucleotides) from $\sim 2,000$ viral species. We excluded sequences from bacteriophage, plant viruses, viral families infecting only invertebrates and endogenous retroviruses. We included viruses that could have both vertebrate and invertebrate hosts, such as vertebrate viruses with insect vectors. Exogenous retroviruses were represented but murine leukemia viruses were removed since they can cross enrich endogenous retroviruses which can represent large portions of vertebrate genomes and mask rarer viral sequences. Control oligonucleotides such as those from human genes, yeast intergenic sequences and human papilloma virus sequences present in HeLa cells were also removed. In all, 92 70-mer oligonucleotides covering (spaced end-to-end) the entire $\mathrm{pol}$ and $\mathrm{gB}$ genes of Equine herpesvirus 1 (EHV-1) were included as PCR screening of water samples indicated they were positive for this virus (Dayaram et al., 2021). The resulting 13,532 oligonucleotides were examined for repetitive elements, short repeats and low complexity regions using RepeatMasker. Repetitive motifs were identified in 234 oligonucleotides, which were removed. The final panel consisted of 13,298 unique sequences which were synthesized (as a panel of biotinylated RNAs) by MYcroarray.

\section{6 | Viral enrichment strategy and sequencing}

In-solution target enrichment via hybridization-based capture was performed according to the manufacturer's protocol (MYbaits ${ }^{\circledR}$ custom targeted enrichment, MYcroarray), with the following modifications: $50 \mu \mathrm{l}$ Dynabeads ${ }^{\circledR} \mathrm{M}-270$ Streptavidin beads (Invitrogen) instead of $30 \mu$ l Dynabeads ${ }^{\circledR}$ MyOne ${ }^{\mathrm{TM}}$ Streptavidin C1 (Invitrogen); hybridization, bead-bait binding and wash steps at $60^{\circ} \mathrm{C} ; 48 \mathrm{hr}$ hybridization time; $200 \mathrm{ng}$ baits per reaction; and $10 \mu \mathrm{l}$ indexed library inputs. Libraries generated from pooled leeches consisting of more than 16 individuals were captured individually, whereas libraries generated from pools of fewer individuals were combined to have a comparable number (15-20) of leeches per capture. This was done to ensure that each individual leech represented in each library was allocated enough bait. Water and sediment samples were pooled in groups of two, with sediment and water pooled separately. Per pooled sample, $500 \mathrm{ng}$ of baits were used to ensure enough bait for each sample. The enriched libraries were re-amplified as described in Alfano et al. (2016). The re-amplified enriched libraries were purified using the MinElute PCR Purification Kit (Qiagen), quantified using the 2200 TapeStation (Agilent Technologies) on D1000 ScreenTapes and pooled in equimolar amounts for single-read sequencing on two lanes of an Illumina NextSeq 500 with the TG NextSeq ${ }^{\circledR} 500 / 550$ High Output Kit v2 (300 cycles).

\section{7 | Data analysis and bioinformatics pipelines}

A total of $219,580,903$ single-end reads 300 bp long were generated (average: 3,181,781 single reads per sample; standard deviation [SD]: 1,481,098; Table S1) and sorted by dual index sequences. Cutadapt v1.16 and Trimmomatic v0.36 were used to remove adapter sequences and low-quality reads using a quality cutoff of 20 and a minimal read length of 30 nt. After trimming, $97 \%$ of the sequences were retained. Leech reads were removed from the dataset by alignment to the Helobdella robusta genome 1.0 (assembly GCA_000326865.1), the only complete genome of Hirudinea available in GenBank at the time of the analyses, and all GenBank leech sequences (4,957 sequences resulting from 'Hirudinea' search) using Bowtie2 v2.3.5.1 (Langmead \& Salzberg, 2012). This left $81 \%$ of the original reads (Table S1). Then, rRNA reads were removed using SortMeRNA (Kopylova et al., 2012), leaving 75\% of the original reads (Table S1). The filtered reads were de novo assembled using both Spades v3.11.1 (Bankevich et al., 2012) and Trinity v2.6.6 (Grabherr et al., 2011) assemblers. The obtained contigs were pooled and clustered to remove duplicated or highly similar sequences using USEARCH v11.0.667 (Edgar, 2010) with a $90 \%$ threshold identity value. The centroids were then subjected to sequential BLAST searches against the NCBI RefSeq viral nucleotide (blastn, 1e-5 E-value threshold) and protein (blastx, 1e-3) databases to identify candidate viral sequences. These candidates were aligned to the complete NCBI nucleotide (blastn, 1e-10) and protein (blastx, 1e-3) databases to identify sequences with higher similarity to non-viral than to viral sequences. In parallel, the adaptor and quality trimmed data were uploaded to Genome Detective (Vilsker et al., 2019), a web base software that assembles viral genomes.

Bacteriophage, invertebrate viruses and retroviruses were excluded from subsequent steps. If our pipeline and Genome Detective generated viral contigs with overlapping sequences, the longest contig was selected for analysis. Filtered reads were mapped to the viral contigs to calculate viral read numbers for each virus. Finally, the viral contigs were mapped to the reference genome of the virus corresponding to the best BLAST hit using Geneious v11.0.2 (Biomatters, Inc.). Baits were also mapped to the same references for each virus. 


\section{8 | Phylogenetic analyses}

Viral contigs were assigned to viral families according to the BLAST results. Representative sequences from these viral families were retrieved from GenBank and aligned with the contigs using MAFFT v7.450 (Katoh \& Standley, 2013). Phylogenetic analysis was performed using the maximum-likelihood method based on the general time reversible substitution model with among-site rate heterogeneity modelled by the $\Gamma$ distribution and estimation of proportion of invariable sites available in RAxML v8 (Stamatakis, 2014), including 500 bootstrap replicates to determine node support. Phylogenetic analyses were performed only on viral contigs (a) showing divergence from known viruses, that is, with both BLAST identity and coverage to the best reference below $95 \%$, to place them into a phylogenetic context and (b) mapping to phylogenetically relevant genomic regions. Therefore, Circoviridae and Anelloviridae contigs were excluded from phylogenetic analyses as were those identified from water.

\section{9 | Leech vertebrate host assignments}

Host identification of leeches followed an eDNA/iDNA workflow (Axtner et al., 2019). In summary, leech samples were digested and short fragments of the mitochondrial markers $12 \mathrm{~S}, 16 \mathrm{~S}$ and cytochrome B were amplified in four PCR replicates each resulting in 12 PCR replicates per sample. We used a twin-tagging two-step PCR protocol and PCR products were sequenced using an Illumina MiSeq (Axtner et al., 2019). After demultiplexing and read processing, each haplotype was taxonomically assigned to a curated reference database using PROTAX (Somervuo et al., 2016).

\subsection{0 | Viral detection confirmation by PCR}

The PCR primers listed in Table S2 were designed to confirm the viral contig sequences generated from the leech samples. For PCRs targeting RNA viruses, $50 \mu$ l of extract was digested with rDNase I (Ambion) following the manufacturer's protocol. The DNAsedigested extracts were then purified using the RNeasy MinElute Cleanup Kit (Qiagen). RNA was reverse transcribed into cDNA using iScript $^{\mathrm{TM}}$ Reverse Transcription Supermix (Bio-Rad). Sediment and water samples that tested positive for EHV and JSRV were screened using previously described pan-herpes and JSRV PCRs (Dayaram et al., 2017; Palmarini et al., 2000). Resulting amplicons were Sanger sequenced.

\subsection{1 | Identification of endogenous viral elements (EVEs)}

To check whether the identified viral contigs represent EVEs of leeches or vertebrate hosts, they were aligned to selected leech and vertebrate genomes (Table S3) using BLAST (blastn, 1e-10 E-value and $50 \%$ query coverage thresholds). Since genome sequences are not available for the leech species and for most host species identified from the bloodmeals, we used the most closely related genomes available in GenBank.

\subsection{2 | Shotgun sequencing}

A subset of 64 leech samples were shotgun sequenced to determine viral enrichment obtained by our capture system. An aliquot of precapture amplified genomic library of each leech sample was taken, pooled in equimolar amounts and sequenced on an Illumina MiSeq with Reagent Kit v3 (150 cycles). The shotgun reads were mapped to the viral contigs obtained by viral capture using Bowtie2 v2.3.5.1 (Langmead \& Salzberg, 2012).

\section{3 | RESULTS}

\section{1 | Positive and negative controls}

In the viral capture control experiment using medicinal leeches fed with virus-spiked blood, all four viruses were detected, with a percentage of on-target viral reads varying among viruses (HAdV> $\mathrm{MeV}>\mathrm{BHV}>$ InfA; Figure 1) according to the viral concentrations measured as $\mathrm{Ct}$ values by qPCR (25 for HAdV, 28 for MeV, 29 for $\mathrm{BHV}$ and 33 for $\operatorname{InfA}$ ). An adeno-associated virus (AAV), which may have been present in the HeLa cell line where the HAdV for the spike-in was cultivated, was also recovered. Most viral contigs coincided with the baits (Figure 1). However, several BHV genomic regions covered by baits were not retrieved, and we also recovered HAdV contigs corresponding to genomic regions not targeted by baits. These regions might have been recovered by baits targeting less closely related adenoviruses or designed for other viruses.

No viral contigs were identified in the negative controls included to monitor laboratory contaminations for either the leech or water experiments. Further potential contamination from laboratory reagents was excluded (see Supporting Information).

\subsection{Leech viral identification}

Viruses were identified in 39 of the 68 leech pools analysed $(57 \%$; Figure 2; Table S4). In 18 of these (46\%), two to three viruses were identified. Sequence data from five vertebrate-infecting viral families were detected. The most common viral group was Rhabdoviridae found in $37 \%$ of samples, followed by Coronaviridae which was identified in $24 \%$ of samples. Members of the Anelloviridae were identified in $12 \%$ of samples, and Parvoviridae and Circoviridae in $3 \%$.

Rhabdoviridae contigs were genetically similar to three viral genera (Table S4). Five contigs were most similar (69\%-77\% nt id) to the Vesicular stomatitis Indiana virus (VSIV; genus Vesiculovirus) 


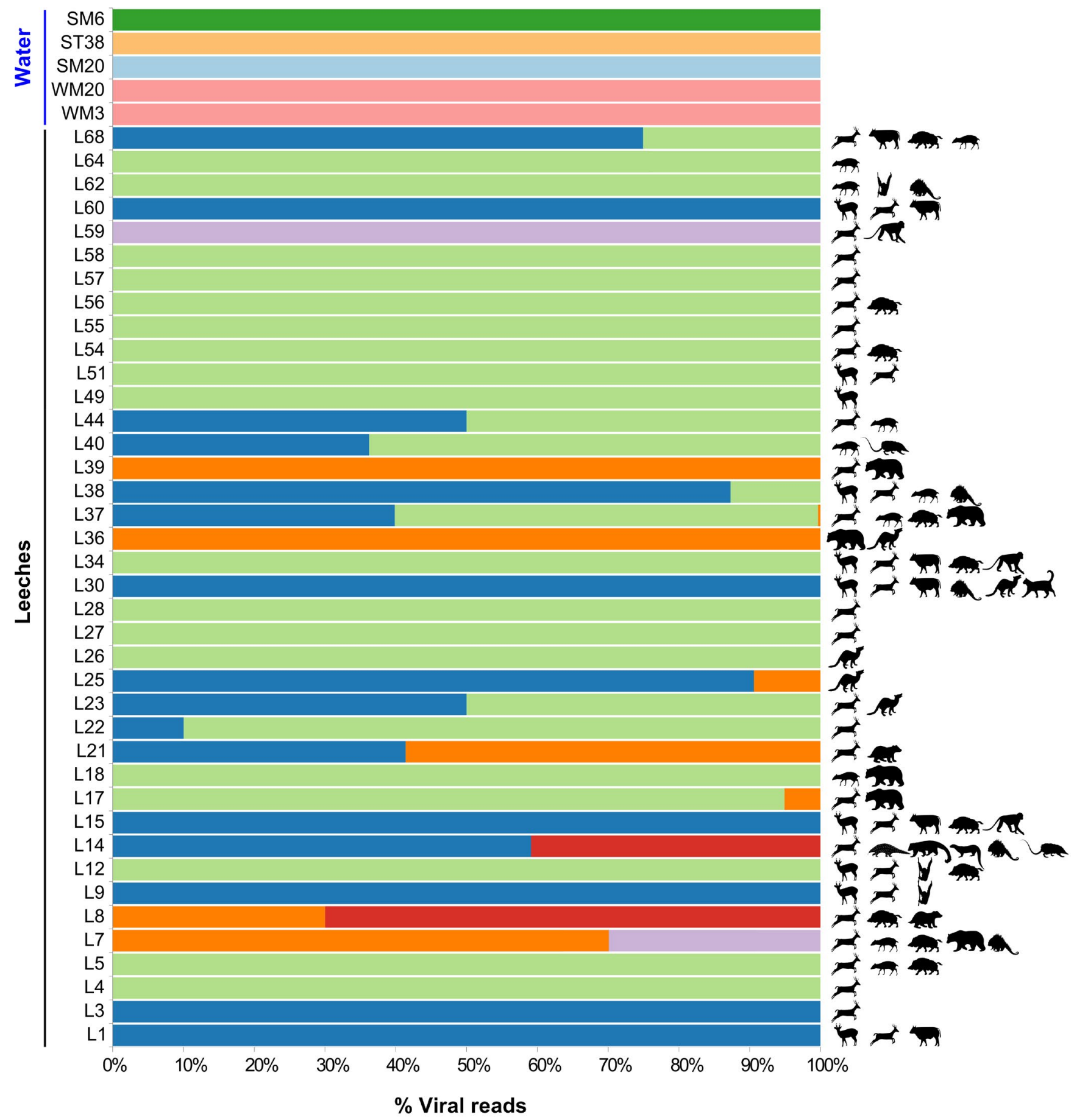

\section{$\begin{array}{ll}\stackrel{\text { 을 }}{\bar{\pi}} & \text { Adenoviridae } \\ \stackrel{0}{y} & \text { Anelloviridae }\end{array}$}

Coronaviridae

Parvoviridae

Herpesviridae

Papillomaviridae

Retroviridae

Rhabdoviridae
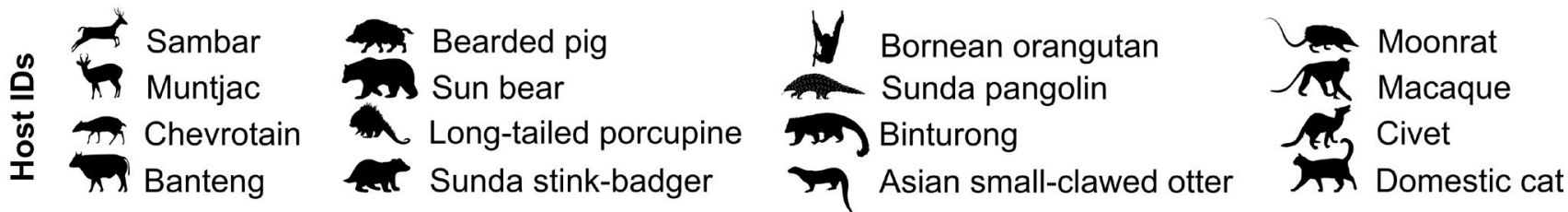

FIGURE 2 Relative abundance of viruses from each family, shown as the percentage of the total number of viral reads in each leech and waterhole sample. For sample names, $\mathrm{S}$ stands for sediment, $\mathrm{W}$ for water, $\mathrm{T}$ for Tanzania, $\mathrm{M}$ for Mongolia and $\mathrm{L}$ for leeches. The leech host assignment for each leech sample is shown on the right (see Table S4 for further details) 
as determined by BLAST searches. The limited similarity of these sequences to known rhabdoviruses suggests they may represent a new genus related to fish rhabdoviruses (Perhabdovirus and Sprivirus) or Vesiculovirus (Figure 3A; Figure S1). The other contigs clustered phylogenetically, suggesting they represent two new species of a rhabdovirus related to lyssaviruses (Figure 3A; Figure S1). Although in most cases one contig per sample was observed, in five samples (L4, L12, L23, L58 and L68) two different viruses were found. Most of the oligonucleotide baits were specific for the $L$ gene which encodes the RNA-dependent RNA polymerase. All recovered contigs mapped to the $L$ gene (Figure S2A-C). The viral contig sequences were confirmed by PCR and Sanger sequencing for L55 and L58 (Figure S2D).

All Coronaviridae contigs were most similar to a bat betacoronavirus (70\%-73\% nt id; Table S4). The resulting sequence did not cluster in any of the four clades representing the known Coronaviridae genera, suggesting it may represent a novel coronavirus genus (Figure 3B; Figure S3). Each contig overlapped with the coronavirus RNA-dependent RNA polymerase gene (orf1ab), the main viral region targeted by the baits (Figure S4).

Anelloviridae contigs matched porcine torque teno virus (PTTV; 95\%-96\% nt id), a giant panda anellovirus (GpAV; 81\%-92\% nt id) or a masked palm civet torque teno virus (PI-TTV; $83 \%-92 \%$ nt id;
Table S4). The PTTV contigs were found in two samples (L8 and L37), while the GpAV and PI-TTV contigs were detected in six samples. GpAV was the best match in four samples (L7, L17, L36 and L39) and PI-TTV in three (L21, L25 and L39). In sample L39, both were identified. Every Anelloviridae contig mapped to the non-coding region of the relative reference genome since all Anelloviridae baits targeted the same untranslated region (Figure S5A,C,E). The non-coding region sequenced is not phylogenetically informative and, therefore, phylogenetic analysis was not performed. Viral contigs were confirmed by PCR and Sanger sequencing for samples L7, L17, L25 and L37 (Figure S5B,D,F).

Three Circoviridae contigs matching a porcine circovirus (PCV; $100 \% \mathrm{nt}$ id) were identified in L7 and L59 (Table S4). Two nonoverlapping but adjacent contigs were retrieved from L7. A single contig overlapping with one of the two contigs determined from L7 was recovered from L59 (Figure S6A). The contigs mapped to the PCV replication protein (Rep), targeted by the Circoviridae baits (Figure S6A). The two overlapping contigs of L7 and L59 were confirmed by PCR and Sanger sequencing (Figure S6B). Since the identity of the contigs with known viral sequences in GenBank was $100 \%$, no phylogenetic analysis was performed.

Parvoviridae contigs with the highest similarity to porcine parvovirus (PPV) were found in L8 (1 contig with 98\% nt id) and L14 (2
(A)

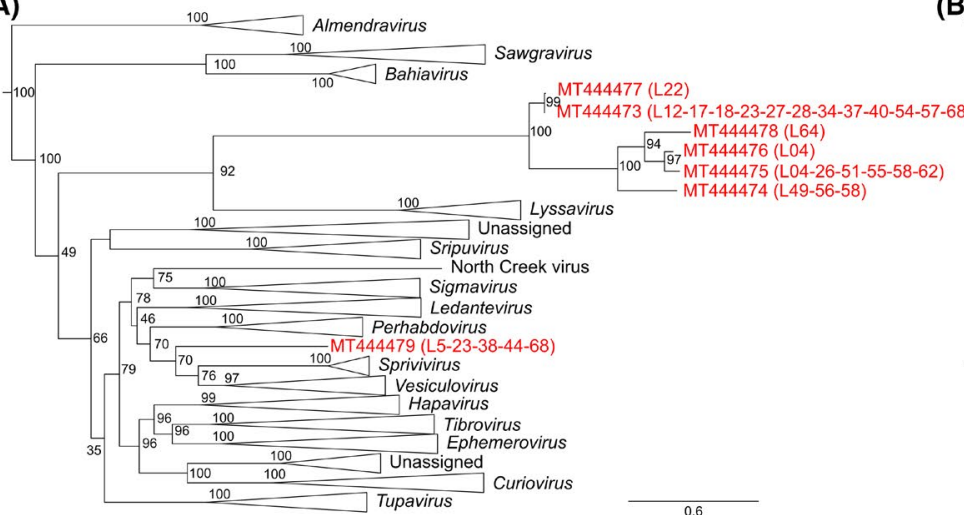

(C)

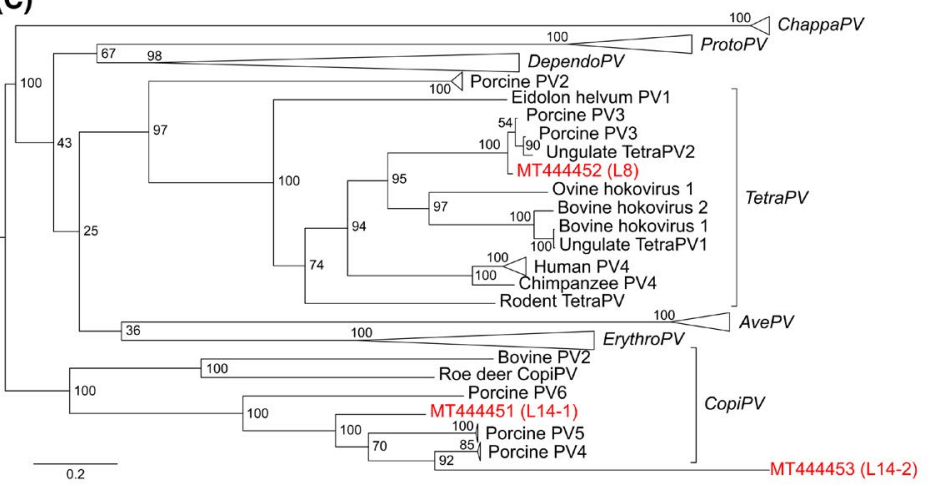

(B)

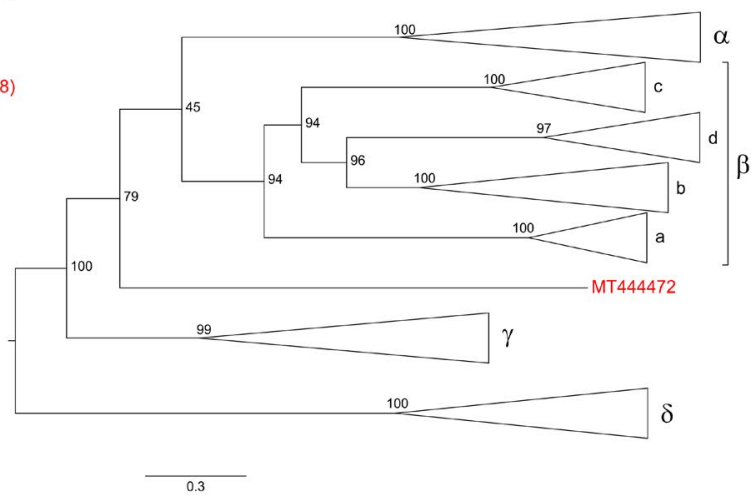

(D)

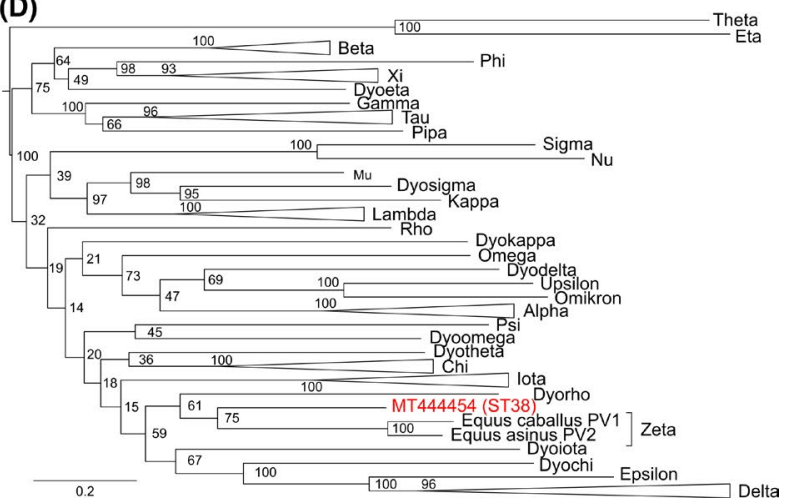

FIGURE 3 Evolutionary relationships of the viral sequence identified in leech and water samples with representatives of the main genera of the Rhabdoviridae (A), Coronaviridae (B), Parvoviridae (C) and Papillomaviridae (D) families. When nearly identical sequences of the same virus were found in multiple samples, consensus sequences were used. The sequences generated in this study are marked in red. B: the four Coronaviridae genera (Alpha-, Beta-, Gamma- and Delta-coronavirus) are indicated, together with the four (a-d) Betacoronavirus subgroups. More details are available in the corresponding supplementary figures 
contigs with $74 \%-77 \%$ nt id; Table S4). The contig of L8 clustered within the Tetraparvovirus genus, close to ungulate parvoviruses (porcine, ovine and bovine PV), while the contigs of L14 within the Copiparvovirus genus, close to PPV4 (Figure 3C; Figure S7). Two of the three contigs mapped to the replicase gene while one from L14 mapped to an intergenic region (Figure $\mathrm{S} 6 \mathrm{C}$ ). Whereas the replicase region of PPV was covered by Parvoviridae baits, the intergenic region was not (Figure S6C). This portion of the virus may have been recovered by other non-Parvoviridae baits.

\section{3 | Viral enrichment}

On-target read proportion was low in both leech and waterholes samples, ranging from $0.00005 \%$ (sample L18) to $0.14 \%$ (sample L58; average $0.01 \%$ ), with a total of 12,654 viral reads out of $149,728,065$ total reads (on-target proportion of $8,5 \mathrm{E}-5$ ) found in 44 (39 leech and 5 waterhole samples) out of 89 total samples tested (68 leech and 21 waterhole samples; Table S5). We compared the results of capture of the leech samples to shotgun sequencing of a subset of 64 samples. In this subset, capture yielded 11,581 viral reads out of a total of $137,171,077$ reads with an on-target proportion of $8,4 \mathrm{E}-5$, whereas four viral reads were found by shotgun sequencing out of $4,747,786$ total reads corresponding to an on-target proportion of $8,4 \mathrm{E}-7$. Viral enrichment by capture resulted in a 100 -fold increase in on-target viral read proportion. In addition, the viral reads obtained by capture from leeches and waterholes were not randomly distributed across the viral genomes but aligned to regions targeted by baits (Figures S2, S4-S6, S8, S9), reaching up to 479X (sample L58) mean depth of coverage in these regions (range 1.2X-479X). This and the 100-fold increase in on-target viral reads strongly indicate that enrichment of the presence of viral genetic material in the samples occurred.

\section{4 | Leech bloodmeal host assignments}

Metabarcoding analysis detected bearded pigs (Sus barbatus) in all samples yielding porcine viruses, such as porcine circovirus (L7), porcine parvovirus (L8) and porcine torque teno virus (L8 and L37). Four leech samples with giant panda anellovirus (L7, L17, L36 and L39) sequences yielded sun bear Helarctos malayanus sequences. Sequences aligning to the Malay civet Viverra tangalunga were identified in one of the three samples (L25) with masked palm civet torque teno virus sequences. In all, 14 of the 16 samples with the potentially new coronavirus genus (87.5\%) yielded deer sequences, specifically sambar Rusa unicolor, suggesting the novel coronavirus might be a cervid virus. Similarly, the novel Lyssavirus-like Rhabdoviridae sequences were associated with cervid species (sambar or Muntiacus sp.; 16 of 22 samples, 73\%). However, due to the high prevalence of deer in the samples tested (70\%), we could not reject that the occurrence of viruses and deer are independent variables $\left(\chi_{\text {Coronaviridae }}^{2}=1.916,1\right.$ $\left.d f, p=0.1663 ; \chi_{\text {Rhabdoviridae }}^{2}=1.046,1 d f, p=0.3064\right)$.

\section{5 | Identification of EVEs}

Retroviridae contigs similar to the simian and feline foamy virus (Spumaretrovirinae subfamily, $79 \%-82 \% \mathrm{nt}$ id) were detected in three samples (L7, L46 and L64) where Tragulus napu was identified as potential leech host species (Table S4). These sequences showed high identity to the Tragulus javanicus genome by BLAST search (Evalue $>1 \mathrm{e}-45$; query coverage $>98 \%$ ). Therefore, we excluded those sequences as potential EVEs. None of the other viral sequences identified had significative blast hit (E-value $>1$-10; query coverage $>50 \%$ ) to the tested leech and vertebrate host genomes. Because of lack of sample material available and sample degradation in the environment and during storage, we could not perform further PCR tests to completely exclude that some of the sequences identified represent EVEs.

\subsection{Waterhole viral identification}

Five waterholes from Tanzania and six from Mongolia were tested. From each waterhole, one water filtrate and one sediment sample were collected (except for one Mongolian waterhole where only sediment was collected), for a total of 21 samples. Five samples (two water and three sediment samples) were positive for viral sequences (23.8\%). In filtered water and sediment, samples collected from the same waterhole, only one virus per sample was identified and in one location (WM20 and SM20) contigs from different viral families were isolated based on sample type. Differences between sediment and water are not unexpected as the sediment likely represents a longerterm accumulation of biomaterial and the water represents more acute contamination at the surface and variable mixing throughout.

Of the 11 water filtrate samples tested, two samples from Mongolia (WM3 and WM20; 18.2\%) had viral contigs with $100 \%$ nt identity to Equid herpesvirus 1 and 3 (EHV-1 and EHV-3). The contig of WM20 mapped to the membrane glycoprotein B, whereas the two contigs of WM3 to the DNA packaging protein and membrane glycoprotein $\mathrm{G}$, all regions covered by the Herpesviridae baits (Figure S8A-D). A nested pan-herpes PCR targeting the DNA polymerase gene and the resulting Sanger sequences further confirmed EHV presence (Figure S8E). Several equine species including domestic horses inhabit the Gobi Desert (Kaczensky et al., 2015), which is consistent with the presence of these viruses. A further study performed hybridization capture on EHV-positive samples using baits tiled across entire EHV genomes, enabling the recovery of the almost entire EHV-1 genome (Dayaram et al., 2021).

Of 12 sediment samples tested, two from Mongolia and one from Tanzania yielded viral sequences $(25 \%)$ representing the Retroviridae, Adenoviridae and Papillomaviridae families. Four contigs mapping to the protease (pro) gene of the Jaagsiekte sheep retrovirus (JSRV; $100 \% \mathrm{nt}$ id) were identified in Mongolian sediment (SM6; Figure S9A). JSRV from SM6 was confirmed by PCR (Figure S9A). Mongolia sediment sample SM20 was positive for Equine adenovirus (100\% nt id) with a contig mapping between the 
pVI and hexon capsid genes (Figure S9B). Multiple equine species are found in the Gobi Desert in Mongolia; therefore, it is likely that the water sources sampled may have been frequented by these species (Nandintsetseg et al., 2016). Sediment sample ST38 from Tanzania was positive for a Zetapapillomavirus related to the Equus caballus papillomavirus and Equus asinus papillomavirus (74\% nt id; E1-E2 genes; Figure 3D; Figures S9C and S10), consistent with the detection of Plains zebra's Equus quagga DNA from this water source (Seeber et al., 2019).

\section{4 | DISCUSSION}

We demonstrate for the first time that both eDNA and iDNA sources can be used to survey known and novel viruses. Many of the viruses identified were highly divergent from available viral reference genomes (homology 45\%-100\%, average 80\%). DNA and RNA viruses could be detected in $57 \%$ and $23.8 \%$ of the iDNA (leech) and eDNA (waterhole) samples, respectively. Congruence of host DNA assignment for leeches and viral families identified suggests that bloodmeals are useful for determining vertebrate viral diversity. Detection of equine viruses from African and Mongolian waterholes, where intense wild equid visitation rates were directly observed, suggests eDNA-derived viruses reflect host utilization of the water rather than other environmental sources such as fomites. While host assignments are difficult to establish for novel viruses from eDNA (multiple hosts sources) and, to a lesser extent, iDNA, the results narrow the possible number of taxa down to a small portion of the overall faunal diversity within the regions examined. For example, the novel coronavirus was associated, though not statistically significantly, with sambar. This suggests targeted sampling and virus-specific PCRs could be used to examine prevalence in the species to establish whether they are a potential viral reservoir. Narrowing down the potential taxa that need to be screened in biodiversity hotspots will be critical, particularly for viruses hosted at low prevalence.

A recent human blood virome study (Moustafa et al., 2017) also demonstrated that not generally blood-associated viruses can be detected in blood. Therefore, leech bloodmeals should not restrict viral detection exclusively to blood-borne viruses. The virome detectable in water depends on host seasonality and faunal geographical distributions determining which species are present in an environment. For example, avian influenza virus detection in aquatic environments is affected by season and site-specific density of water fowl (Densmore et al., 2017). In the current study, water sampling was performed in the dry season, when animals congregate at limited water sources increasing the likelihood of detecting animal pathogens in the water. Animal viruses detected in water are expected to result from contamination from stool, urine (Rohani et al., 2009), nasal exudates and shed saliva. The viruses we identified in water are known respiratory transmitted viruses (EHV-1, equine adenovirus, JSRV) or viruses transmitted through skin abrasions (Equus asinus papillomavirus).
PCR-based approaches have been used to detect known pathogens from flies (Bitome-Essono et al., 2017; Gogarten et al., 2019) or from medicinal leeches under laboratory conditions (Kampmann et al., 2017). While major findings have resulted from such analyses, PCR-based approaches are often poorly suited to the discovery of novel viruses that may be highly divergent as PCRs often fail when viral divergence exceeds $5 \%-10 \%$, particularly relevant to RNA viruses (Schlaberg et al., 2017). The unknown viral diversity in the wild, and the potential degradation of viral nucleic acids in bloodmeals or the environment, may affect PCR detection resulting in high falsenegative rates. Hybridization capture overcomes such limitations because the short baits can capture divergent and degraded DNA. With our capture system, we were able to identify viral sequences with up to 55\% divergence from known viral genomes. The extensive virus representation in the bait set allows for the determination of both viral presence and viral diversity. The ability of oligonucleotides with substantial divergence from the target sequence to capture more distantly related sequences is particularly useful in virology since most viruses are uncharacterized in wildlife and many evolve rapidly (Briese et al., 2015; Howard \& Fletcher, 2012). The overall on-target viral read recovery was low. This was not unexpected since it is unlikely that any bloodmeal was strongly viraemic or that large amounts of virus were shed into water. Nevertheless, our viral capture system generated a 100-fold higher viral enrichment compared to shotgun sequencing. Furthermore, viral enrichment concentrated in regions of the viral genomes where baits were designed, leading to high coverage (up to $479 \mathrm{X}$ ) at these positions. This allowed the assembly of viral genome fragments that could be analysed phylogenetically and further confirmed by PCR.

Using short RNA baits to capture highly conserved sequences from known vertebrate viral genomes is useful and relatively inexpensive for providing an initial viral identification (Figure 1). However, to fully characterize each virus, the RNA oligonucleotide bait set would need to be customized to retrieve full-length viral genomes which has also been done successfully for novel divergent viruses (Alfano et al. 2016). This is a possible strategy to further investigate viruses of interest, whereas initial screening with fulllength genomes for all viruses is currently too costly.

Several novel viruses were identified from leech bloodmeals, which is not unexpected as little is known about the virology of wildlife in Southeast Asia. Several viral contigs were phylogenetically distinct from known viruses and may represent new genera. For example, the novel coronavirus identified in leech bloodmeals did not cluster with any known Coronaviridae clades. We could also tentatively associate the novel corona- and rhabdoviruses with cervids, which are regularly sold as bushmeat in wildlife markets (Nasi et al., 2011). Both recent coronavirus epidemics (SARS-CoV, Drosten et al., 2003 and SARS-CoV-2, Zhou et al., 2020) spilled over from wildlife. This suggests that e/iDNA-based pathogen surveillance approaches may complement efforts to proactively identify novel viruses that could potentially spillover to humans or livestock.

Collecting wild haematophagous invertebrates such as leeches or water and sediments has both advantages and disadvantages 
compared to invasively collected wildlife samples. Large amounts of DNA can be extracted from bloodmeals, in particular when leeches are processed in bulk. We pooled up to 77 leeches and many of our leech bulk samples contained a diverse mix of mammalian DNA. A disadvantage of leeches is that they cannot be found in all environments: for example, haematophagous terrestrial species are restricted to tropical rainforests of Asia, Madagascar and Australia (Schnell et al., 2018). Leech feeding biases could influence diversity surveys (Abrams et al., 2019; Schnell et al., 2015). However, this disadvantage could be overcome in the future by employing additional invertebrates such as mosquitoes ( $\mathrm{Ng}$ et al., 2011) or carrion flies (Hoffmann et al., 2016). Waterholes are commonly found in almost all environments. In environments with seasonal water shortages, DNA from animals can become highly concentrated due to many animals utilizing rare water sources. The disadvantages are that the dilution factor of water, depending on water body size, can obscure rare DNA sequences and mixed host species sequences are generally the rule rather than the exception. RNA degradation can be a limiting factor for the detection of RNA viruses in environmental samples. RNA virus stability depends on many factors such as temperature, $\mathrm{pH}$ and UV exposure (Lowen \& Steel, 2014). We cannot exclude that such factors may have played a role in the lack of detection of RNA viruses (except a retrovirus) in water. In contrast, both RNA and DNA viruses have been shown to be detectable in medical leeches even 50 days after ingestion, despite a gradual decline in concentration over time (Kampmann et al., 2017).

Viral enrichment by hybridization capture from eDNA and its subdiscipline iDNA may be a useful and economical tool for viral identification and characterization prior to potential viral emergence. eDNA and iDNA may be the only option to detect viruses circulating in wild environments where direct access to animals is difficult or highly restricted. The current study suggests this approach will be successful in complementing invasive approaches or replacing them in environments where invasive approaches are impossible.

\section{ACKNOWLEDGEMENTS}

We thank Joseph DeRisi for guidance in using his oligonucleotide data. We thank Peter Seeber and Sanatana-Erini Soilemetzidou for collection of water and sediment samples in Africa and Mongolia, respectively. We thank the Sabah Forestry Department, especially Johnny Kissing, Peter Lagan and Datuk Sam Mannan, for supporting the fieldwork and the Sabah Biodiversity Council for providing research, collection and export permits (JKM/MBS.1000-2/3 JLD.2) for the leech work. This project received financial support from the German Federal Ministry of Education and Research (BMBF FKZ: 01LN1301A) to N.A., J.A., A.M. and A.W. and was supported by funds from the Leibniz Gemeinschaft, SAW-2015-IZW-1 440 to A.D. and A.D.G.

\section{AUTHORS' CONTRIBUTIONS}

A.D.G. and A.W. designed the study; A.M. and S.T.W. collected the leeches in the field; J.A. performed the leech nucleic acids extractions and the PCRs on leeches; N.A. and A.D. performed the capture experiments; N.A. and K.T. performed the bioinformatics and phylogenetic analyses; N.A., A.D., A.D.G. and A.W. wrote the manuscript; N.A., A.D., J.A., K.T., M.-L.K., M.T.P.G., A.W. and A.D.G. reviewed the manuscript. All authors read and approved the final manuscript.

\section{PEER REVIEW}

The peer review history for this article is available at https://publo ns.com/publon/10.1111/2041-210X.13661.

\section{DATA AVAILABILITY STATEMENT}

The dataset generated and analysed during this study is available in the NCBI Sequence Read Archive (SRA) repository under the accession number PRJNA627811 (https://www.ncbi.nlm.nih.gov/ bioproject/PRJNA627811/). The consensus sequences of the viral contigs obtained in this study were deposited in GenBank under the accession numbers MT444451-MT444487. Supplementary data for this publication can be found on Dryad Digital Repository https:// doi.org/10.5061/dryad.2v6wwpznc (Alfano et al., 2021).

\section{ORCID}

Niccolò Alfano (iD https://orcid.org/0000-0002-8174-8568

Anisha Dayaram iD https://orcid.org/0000-0002-9247-9260

Jan Axtner (iD https://orcid.org/0000-0003-1269-5586

Kyriakos Tsangaras (D) https://orcid.org/0000-0001-7454-0454

Marie-Louise Kampmann iD https://orcid.

org/0000-0001-5403-5960

M. Thomas P. Gilbert (iD https://orcid.org/0000-0002-5805-7195

Andreas Wilting (iD https://orcid.org/0000-0001-5073-9186

Alex D. Greenwood iD https://orcid.org/0000-0002-8249-1565

\section{REFERENCES}

Abrams, J. F., Hörig, L. A., Brozovic, R., Axtner, J., Crampton-Platt, A., Mohamed, A., Wong, S. T., Sollmann, R., Yu, D. W., \& Wilting, A. (2019). Shifting up a gear with iDNA: From mammal detection events to standardised surveys. Journal of Applied Ecology, 56(7), 1637-1648.

Alfano, N., Dayaram, A., Axtner, J., Tsangaras, K., Kampmann, M.-L., Mohamed, A., Wong, S. T., Gilbert, M. T., Wilting, A., \& Greenwood, A. D. (2021). Data from: Non-invasive surveys of mammalian viruses using environmental DNA. Dryad Digital Repository, https://doi. org/10.5061/dryad.2v6wwpznc

Alfano, N., Michaux, J., Morand, S., Aplin, K., Tsangaras, K., Löber, U., Fabre, P.-H., Fitriana, Y., Semiadi, G., Ishida, Y., Helgen, K. M., Roca, A. L., Eiden, M. V., \& Greenwood, A. D. (2016). Endogenous gibbon ape leukemia virus identified in a rodent (Melomys burtoni subsp.) from Wallacea (Indonesia). Journal of Virology, 90(18), 8169-8180.

Axtner, J., Crampton-Platt, A., Hörig, L. A., Mohamed, A., Xu, C. C., Yu, D. W., \& Wilting, A. (2019). An efficient and robust laboratory workflow and tetrapod database for larger scale environmental DNA studies. GigaScience, 8(4), giz029. https://doi.org/10.1093/gigascience/ giz029

Bankevich, A., Nurk, S., Antipov, D., Gurevich, A. A., Dvorkin, M., Kulikov, A. S., Lesin, V. M., Nikolenko, S. I., Pham, S., Prjibelski, A. D., Pyshkin, A. V., Sirotkin, A. V., Vyahhi, N., Tesler, G., Alekseyev, M. A., \& Pevzner, P. A. (2012). SPAdes: A new genome assembly algorithm and its applications to single-cell sequencing. Journal of Computational Biology, 19(5), 455-477. https://doi.org/10.1089/cmb.2012.0021

Bitome-Essono, P.-Y., Ollomo, B., Arnathau, C., Durand, P., Mokoudoum, N. D., Yacka-Mouele, L., Okouga, A.-P., Boundenga, L., Mve-Ondo, 
B., Obame-Nkoghe, J., Mbehang-Nguema, P., Njiokou, F., Makanga, B., Wattier, R., Ayala, D., Ayala, F. J., Renaud, F., Rougeron, V. Bretagnolle, F., ... Paupy, C. (2017). Tracking zoonotic pathogens using blood-sucking flies as 'flying syringes'. eLife, 6, e22069. https:// doi.org/10.7554/eLife.22069

Briese, T., Kapoor, A., Mishra, N., Jain, K., Kumar, A., Jabado, O. J., \& Lipkin, W. I. (2015). Virome capture sequencing enables sensitive viral diagnosis and comprehensive virome analysis. Mbio, 6(5), e01491-15.

Chen, E. C., Miller, S. A., DeRisi, J. L., \& Chiu, C. Y. (2011). Using a panviral microarray assay (Virochip) to screen clinical samples for viral pathogens. Journal of Visualized Experiments, (50). https://doi. org $/ 10.3791 / 2536$

Daszak, P., Cunningham, A. A., \& Hyatt, A. D. (2000). Emerging infectious diseases of wildlife-threats to biodiversity and human health. Science, 287(5452), 443-449.

Dayaram, A., Franz, M., Schattschneider, A., Damiani, A. M., Bischofberger, S., Osterrieder, N., \& Greenwood, A. D. (2017). Long term stability and infectivity of herpesviruses in water. Scientific Reports, 7, 46559. https://doi.org/10.1038/srep46559

Dayaram, A., Seeber, P., Courtiol, A., Soilemetzidou, S., Tsangaras, K., Franz, M., McEwen, G., Azab, W., Kaczensky, P., Melzheimer, J., East, M. L., Ganbaatar, O., Walzer, C., Osterrieder, N., \& Greenwood, A. D. (2021). Seasonal host and ecological drivers may promote restricted water as a viral vector. Science of the Total Environment, 773, 145446.

Densmore, C. L., Iwanowicz, D. D., Ottinger, C. A., Hindman, L. J., Bessler, A., Iwanowicz, L. R., Prosser, D. J., Whitbeck, M., \& Driscoll, C. P. (2017). Molecular detection of avian influenza virus from sediment samples in waterfowl habitats on the Delmarva Peninsula, United States. Avian Diseases, 61(4), 520-525.

Drosten, C., Günther, S., Preiser, W., van der Werf, S., Brodt, H.-R., Becker, S., Rabenau, H., Panning, M., Kolesnikova, L., Fouchier, R. A. M., Berger, A., Burguière, A.-M., Cinatl, J., Eickmann, M., Escriou, N., Grywna, K., Kramme, S., Manuguerra, J.-C., Müller, S., ... Doerr, H. W. (2003). Identification of a novel coronavirus in patients with severe acute respiratory syndrome. New England Journal of Medicine, 348(20), 1967-1976. https://doi.org/10.1056/NEJMoa030747

Edgar, R. C. (2010). Search and clustering orders of magnitude faster than BLAST. Bioinformatics, 26(19), 2460-2461. https://doi.org/10.1093/ bioinformatics/bta461

Gogarten, J. F., Düx, A., Mubemba, B., Pléh, K., Hoffmann, C., Mielke, A., Müller-Tiburtius, J., Sachse, A., Wittig, R. M., Calvignac-Spencer, S., \& Leendertz, F. H. (2019). Tropical rainforest flies carrying pathogens form stable associations with social nonhuman primates. Molecular Ecology, 28(18), 4242-4258. https://doi.org/10.1111/mec.15145

Grabherr, M. G., Haas, B. J., Yassour, M., Levin, J. Z., Thompson, D. A., Amit, I., Adiconis, X., Fan, L., Raychowdhury, R., Zeng, Q., Chen, Z., Mauceli, E., Hacohen, N., Gnirke, A., Rhind, N., di Palma, F., Birren, B. W., Nusbaum, C., Lindblad-Toh, K., ... Regev, A. (2011). Trinity: Reconstructing a full-length transcriptome without a genome from RNA-Seq data. Nature Biotechnology, 29(7), 644.

Hoffmann, C., Stockhausen, M., Merkel, K., Calvignac-Spencer, S., \& Leendertz, F. H. (2016). Assessing the feasibility of fly based surveillance of wildlife infectious diseases. Scientific Reports, 6(1), 1-9. https://doi.org/10.1038/srep37952

Howard, C. R., \& Fletcher, N. F. (2012). Emerging virus diseases: Can we ever expect the unexpected? Emerging Microbes \& Infections, 1(1), 1-9.

Hoye, B. J., Munster, V. J., Nishiura, H., Klaassen, M., \& Fouchier, R. A. (2010). Surveillance of wild birds for avian influenza virus. Emerging Infectious Diseases, 16(12), 1827. https://doi.org/10.3201/eid1612.100589

Johnson, J., Howard, K., Wilson, A., Ward, M., Gilbert, G. L., \& Degeling, C. (2019). Public preferences for One Health approaches to emerging infectious diseases: A discrete choice experiment. Social Science \& Medicine, 228, 164-171. https://doi.org/10.1016/j.socsc imed.2019.03.013
Jones, K. E., Patel, N. G., Levy, M. A., Storeygard, A., Balk, D., Gittleman, J. L., \& Daszak, P. (2008). Global trends in emerging infectious diseases. Nature, 451(7181), 990-993. https://doi.org/10.1038/nature06536

Kaczensky, P., Lkhagvasuren, B., Pereladova, O., Hemami, M., \& Bouskila, A. (2015). Equus hemionus. The IUCN Red List of Threatened Species 2015: e. T7951A45171204.

Kampmann, M.-L., Schnell, I. B., Jensen, R. H., Axtner, J., Sander, A. F., Hansen, A. J., Bertelsen, M. F., Greenwood, A. D., Gilbert, M. T. P., \& Wilting, A. (2017). Leeches as a source of mammalian viral DNA and RNA-A study in medicinal leeches. European Journal of Wildlife Research, 63(2), 36. https://doi.org/10.1007/s10344-017-1093-6

Katoh, K., \& Standley, D. M. (2013). MAFFT multiple sequence alignment software version 7: Improvements in performance and usability. Molecular Biology and Evolution, 30(4), 772-780. https://doi. org/10.1093/molbev/mst010

Kopylova, E., Noé, L., \& Touzet, H. (2012). SortMeRNA: Fast and accurate filtering of ribosomal RNAs in metatranscriptomic data. Bioinformatics, 28(24), 3211-3217. https://doi.org/10.1093/bioin formatics/bts 611

Langmead, B., \& Salzberg, S. (2012). Fast gapped-read alignment with bowtie 2. Nature Methods, 9(4), 357-359. PMID: 22388286.

Leroy, E. M., Epelboin, A., Mondonge, V., Pourrut, X., Gonzalez, J.P., Muyembe-Tamfum, J.-J., \& Formenty, P. (2009). Human Ebola outbreak resulting from direct exposure to fruit bats in Luebo, Democratic Republic of Congo, 2007. Vector-Borne and Zoonotic Diseases, 9(6), 723-728.

Lowen, A. C., \& Steel, J. (2014). Roles of humidity and temperature in shaping influenza seasonality. Journal of Virology, 88(14), 7692-7695.

Meyer, M., \& Kircher, M. (2010). Illumina sequencing library preparation for highly multiplexed target capture and sequencing. Cold Spring Harbor Protocols, 2010(6), pdb.prot5448. https://doi.org/10.1101/ pdb.prot5448

Mosher, B. A., Huyvaert, K. P., Chestnut, T., Kerby, J. L., Madison, J. D., \& Bailey, L. L. (2017). Design-and model-based recommendations for detecting and quantifying an amphibian pathogen in environmental samples. Ecology and Evolution, 7(24), 10952-10962. https://doi. org/10.1002/ece3.3616

Moustafa, A., Xie, C., Kirkness, E., Biggs, W., Wong, E., Turpaz, Y., Bloom, K., Delwart, E., Nelson, K. E., Venter, J. C., \& Telenti, A. (2017). The blood DNA virome in 8,000 humans. PLoS Path, 13(3), e1006292.

Nandintsetseg, D., Kaczensky, P., Ganbaatar, O., Leimgruber, P., \& Mueller, T. (2016). Spatiotemporal habitat dynamics of ungulates in unpredictable environments: The khulan (Equus hemionus) in the Mongolian Gobi desert as a case study. Biological Conservation, 204, 313-321.

Nasi, R., Taber, A., \& Van Vliet, N. (2011). Empty forests, empty stomachs? Bushmeat and livelihoods in the Congo and Amazon Basins. International Forestry Review, 13(3), 355-368. https://doi. org/10.1505/146554811798293872

Ng, T. F. F., Willner, D. L., Lim, Y. W., Schmieder, R., Chau, B., Nilsson, C., Anthony, S., Ruan, Y., Rohwer, F., \& Breitbart, M. (2011). Broad surveys of DNA viral diversity obtained through viral metagenomics of mosquitoes. PLoS ONE, 6(6), e20579. https://doi.org/10.1371/journ al.pone.0020579

Palmarini, M., Datta, S., Omid, R., Murgia, C., \& Fan, H. (2000). The long terminal repeat of Jaagsiekte sheep retrovirus is preferentially active in differentiated epithelial cells of the lungs. Journal of Virology, 74(13), 5776-5787.

Peeri, N. C., Shrestha, N., Rahman, M. S., Zaki, R., Tan, Z., Bibi, S., Baghbanzadeh, M., Aghamohammadi, N., Zhang, W., \& Haque, U. (2020). The SARS, MERS and novel coronavirus (COVID-19) epidemics, the newest and biggest global health threats: What lessons have we learned? International Journal of Epidemiology, 49(3), 717-726. https://doi.org/10.1093/ije/dyaa033

Pruvot, M., Khammavong, K., Milavong, P., Philavong, C., Reinharz, D., Mayxay, M., Rattanavong, S., Horwood, P., Dussart, P., 
Douangngeun, B., Theppangna, W., Fine, A. E., Olson, S. H., Robinson, M., \& Newton, P. (2019). Toward a quantification of risks at the nexus of conservation and health: The case of bushmeat markets in Lao PDR. Science of the Total Environment, 676, 732-745. https://doi. org/10.1016/j.scitotenv.2019.04.266

Rohani, P., Breban, R., Stallknecht, D. E., \& Drake, J. M. (2009). Environmental transmission of low pathogenicity avian influenza viruses and its implications for pathogen invasion. Proceedings of the National Academy of Sciences of the United States of America, 106(25), 10365-10369. https://doi.org/10.1073/pnas.0809026106

Schlaberg, R., Queen, K., Simmon, K., Tardif, K., Stockmann, C., Flygare, S., Kennedy, B., Voelkerding, K., Bramley, A., Zhang, J., Eilbeck, K., Yandell, M., Jain, S., Pavia, A. T., Tong, S., \& Ampofo, K. (2017). Viral pathogen detection by metagenomics and pan-viral group polymerase chain reaction in children with pneumonia lacking identifiable etiology. The Journal of Infectious Diseases, 215(9), 1407-1415.

Schnell, I. B., Bohmann, K., Schultze, S. E., Richter, S. R., Murray, D. C., Sinding, M.-H., Bass, D., Cadle, J. E., Campbell, M. J., Dolch, R., Edwards, D. P., Gray, T. N. E., Hansen, T., Hoa, A. N. Q., Noer, C. L., Heise-Pavlov, S., Sander Pedersen, A. F., Ramamonjisoa, J. C., Siddall, M. E., ... Gilbert, M. T. P. (2018). Debugging diversity-A pancontinental exploration of the potential of terrestrial blood-feeding leeches as a vertebrate monitoring tool. Molecular Ecology Resources, 18(6), 1282-1298. https://doi.org/10.1111/1755-0998.12912

Schnell, I. B., Sollmann, R., Calvignac-Spencer, S., Siddall, M. E., Douglas, W. Y., Wilting, A., \& Gilbert, M. T. P. (2015). iDNA from terrestrial haematophagous leeches as a wildlife surveying and monitoring tool-prospects, pitfalls and avenues to be developed. Frontiers in Zoology, 12(1), 24. https://doi.org/10.1186/s12983-015-0115-z

Seeber, P. A., McEwen, G. K., Löber, U., Förster, D. W., East, M. L., Melzheimer, J., \& Greenwood, A. D. (2019). Terrestrial mammal surveillance using hybridization capture of environmental DNA from African waterholes. Molecular Ecology Resources, 19(6), 1486-1496. https://doi.org/10.1111/1755-0998.13069

Sharp, P. M., \& Hahn, B. H. (2011). Origins of HIV and the AIDS pandemic. Cold Spring Harbor Perspectives in Medicine, 1(1), a006841. https:// doi.org/10.1101/cshperspect.a006841

Śmietanka, K., Woźniakowski, G., Kozak, E., Niemczuk, K., Frączyk, M., Bocian, Ł., Kowalczyk, A., \& Pejsak, Z. (2016). African swine fever epidemic, Poland, 2014-2015. Emerging Infectious Diseases, 22(7), 1201. https://doi.org/10.3201/eid2207.151708

Somervuo, P., Koskela, S., Pennanen, J., Henrik Nilsson, R., \& Ovaskainen, O. (2016). Unbiased probabilistic taxonomic classification for DNA barcoding. Bioinformatics, 32(19), 2920-2927. https://doi. org/10.1093/bioinformatics/btw346

Stamatakis, A. (2014). RAxML version 8: A tool for phylogenetic analysis and post-analysis of large phylogenies. Bioinformatics, 30(9), 13121313. https://doi.org/10.1093/bioinformatics/btu033
Swift, L., Hunter, P. R., Lees, A. C., \& Bell, D. J. (2007). Wildlife trade and the emergence of infectious diseases. EcoHealth, 4(1), 25.

Tilker, A., Abrams, J. F., Nguyen, A., Hörig, L., Axtner, J., Louvrier, J., Rawson, B. M., Quang, H. A. N., Guegan, F., Nguyen, T. B., Le, M., Sollmann, R., \& Wilting, A. (2020). Identifying conservation priorities in a defaunated tropical biodiversity hotspot. Diversity and Distributions, 26(4), 426-440.

Vilsker, M., Moosa, Y., Nooij, S., Fonseca, V., Ghysens, Y., Dumon, K., Pauwels, R., Alcantara, L. C., Vanden Eynden, E., Vandamme, A.-M., Deforche, K., \& de Oliveira, T. (2019). Genome Detective: An automated system for virus identification from high-throughput sequencing data. Bioinformatics, 35(5), 871-873. https://doi.org/10.1093/ bioinformatics/bty695

Wang, D., Coscoy, L., Zylberberg, M., Avila, P. C., Boushey, H. A., Ganem, D., \& DeRisi, J. L. (2002). Microarray-based detection and genotyping of viral pathogens. Proceedings of the National Academy of Sciences of the United States of America, 99(24), 15687-15692.

Wilking, H., Ziller, M., Staubach, C., Globig, A., Harder, T. C., \& Conraths, F. J. (2009). Chances and limitations of wild bird monitoring for the avian influenza virus $\mathrm{H} 5 \mathrm{~N} 1-$ Detection of pathogens highly mobile in time and space. PLoS ONE, 4(8), e6639. https://doi.org/10.1371/ journal.pone.0006639

Yozwiak, N. L., Skewes-Cox, P., Stenglein, M. D., Balmaseda, A., Harris, E., \& DeRisi, J. L. (2012). Virus identification in unknown tropical febrile illness cases using deep sequencing. PLoS Neglected Tropical Diseases, 6(2), e1485. https://doi.org/10.1371/journal.pntd.0001485

Zhou, P., Yang, X.-L., Wang, X.-G., Hu, B., Zhang, L., Zhang, W., Si, H.-R., Zhu, Y., Li, B., Huang, C.-L., Chen, H.-D., Chen, J., Luo, Y., Guo, H., Jiang, R.-D., Liu, M.-Q., Chen, Y., Shen, X.-R., Wang, X. I., ... Shi, Z.L. (2020). A pneumonia outbreak associated with a new coronavirus of probable bat origin. Nature, 1-4. https://doi.org/10.1038/s4158 6-020-2012-7

\section{SUPPORTING INFORMATION}

Additional supporting information may be found online in the Supporting Information section.

How to cite this article: Alfano, N., Dayaram, A., Axtner, J., Tsangaras, K., Kampmann, M.-L., Mohamed, A., Wong, S. T., Gilbert, M. T. P., Wilting, A., \& Greenwood, A. D. (2021).

Non-invasive surveys of mammalian viruses using environmental DNA. Methods in Ecology and Evolution, 00, 1-12. https://doi.org/10.1111/2041-210X.13661 